\title{
Commentary
}

\section{Commentary on Misconceptions and misuse of the MMPI-2 in assessing personal injury claimants}

\author{
Paul R. Lees-Haley \\ 2915 Bob Wallace Ave., Huntsville, AL 35805, USA \\ E-mail: paulLH@Lees-Haley.com
}

From a forensic perspective, the 2001 paper by Senior and Douglas on misconceptions and misuse of the MMPI-2 in assessing personal injury claimants signals the most important project concerning the MMPI-2 to appear since the new version was published in 1989. Based on a detailed analysis of protocols from 2441 forensic cases from Brisbane, Australia, Senior and Douglas argue that the traditional approach to MMPI-2 interpretation can not be empirically supported in the forensic context. They are particularly critical of code type interpretations and the use of the MMPI-2 as a basis for diagnostic decisions.

Numerous previous researchers have raised important questions about the use of the MMPI-2 (e.g., see [3, $4,6,8,9,14]$. However, none have addressed the questions in this paper with such a sizeable sample and in such detail, or applied such findings to the forensic neuropsychology context with such forceful and persuasive arguments.

The implications of Senior and Douglas's paper and the larger project of which it is a part affect several dimensions of forensic work at fundamental levels. Three overlapping aspects of differential diagnosis illustrate these problems. These are (1) identification of the most likely diagnosis, (2) ruling out psychological confounders of cognitive measures, and (3) the impact of a sensational weak link on the apparent reliability and validity of our test batteries.

Differential diagnosis, although based on DSM criteria (DSM-IV as of this writing [1]), is routinely affected by test findings because test results affect the judgment of diagnosticians. For example, even if the patient's self report is not completely consistent with the DSM criteria, it would be an unusual clinician who completely ignored powerful test evidence that appears to be valid. Because the MMPI-2 is more widely used than any other test for the psychological aspects of our diagnoses, as distinct from the cognitive measures associated with brain injury, it may have more effect than any other psychological test $[7,10]$.

Ruling out confounders is a routine and essential component of forensic neuropsychological evaluations in which the expert addresses questions of causation, which includes the majority of forensic cases. It is commonplace in forensic neuropsychological reports to find expressions of concern about psychological or functional influences on our cognitive findings, e.g., depression, anxiety, or malingering. But if the potential confounder is illusory, we may not give full credence to valid results.

Finally, in forensic neuropsychology we are subject to challenges over the scientific validity and reliability of our work not only in scientific forums but also in courts. For example, in the United States, the Daubert case and its progeny have inspired scientific challenges to expert methodology in Federal Court and many state courts (Daubert v. Merrell Dow Pharmaceuticals, Inc., 113 S.Ct. 2786 (1993)). Some courts have noted that a chain of scientific reasoning is only as strong as its weakest link. At times this is completely appropriate; at other times, an expert may make an error formulating one conclusion without undermining other conclusions. However, courts and experts may react with skepticism toward the rest of an assessment if one important component is seriously flawed. This skepticism at times may be cosmetic in nature, i.e., the speculative and overly broad assumption that an expert who makes one important error cannot be trusted to do anything right, but it may have serious effects on the credibility of the expert's conclusions in general. 
In conclusion, this article by Senior and Douglas is one of a series of steps in a research undertaking that compels our attention $[2,5,12,13]$. Their work will be attacked not only by individuals whose personal experience convinces them that the MMPI-2 is valid and reliable but also by those with economic interests in maintaining the status quo. It is essential for proponents of psychology as a science to consider their research in a dispassionate manner and follow the data where they lead.

\section{References}

[1] American Psychiatric Association, Diagnostic and statistical manual of mental disorders, (4th ed.), Author, Washington, DC, 1994.

[2] W. Baker, G. Senior and L. Douglas, Difficulty in classifying medicolegal groups with the MMPI-2. Poster presented at the 19th Annual Conference of the National Academy of Neuropsychology, San Antonio, Texas, USA, 10-13 November 1999.

[3] W.G. Dahlstrom, Comparability of two-point high-point code patterns from original MMPI norms to MMPI-2 norms for the restandardization sample, Journal of Personality Assessment 59 (1992), 153-164.

[4] D.W.Edwards, T.L. Morrison and H.N. Weissman, The MMPI and MMPI-2 in an outpatient sample: Comparisons of code types, validity scales, and clinical scales, Journal of Personality Assessment 61 (1993), 1-18.

[5] J.R. Henry and G. Senior, Examining the diagnostic utility of MMPI-2 scales in personal injury cases. Poster presented at the 1st Annual Conference of the APS College of Forensic Psychologists, Sydney, NSW, Australia, 7-11 February 2001.

[6] D.H. Humphrey AND W.G. Dahlstrom, The impact of changing from the MMPI to the MMPI-2 on profile configurations, Journal of Clinical \& Experimental Neuropsychology 17 (1995), 58-64.

[7] P.R. Lees-Haley, H.H. Smith, C.W. Williams and J.T. Dunn, Forensic neuropsychological test usage: An empirical survey, Archives of Clinical Neuropsychology 11(1) (1996), 45-51.

[8] H.B. Miller AND C.E. Paniak, MMPI and MMPI-2 profile and code type congruence in a brain-injured sample, Journal of Clinical Psychology 50 (1994), 361-367.

[9] P.H. Munley, A comparison of MMPI-2 and MMPI T-scores for men and women, Journal of Clinical Psychology 47 (1991), 87-91.

[10] K.S. Pope, J.N. Butcher and J. Seelen, The MMPI, MMPI-2, \& MMPI-A in court, American Psychological Association, Washington, DC, 1993, 2000.

[11] G. Senior and L. Douglas, Misconceptions and misuse of the MMPI-2 in assessing personal injury claimants, NeuroRehabilitation 16 (2001), 203-213.

[12] G. Senior, J. Vlahov and R. Shapcott, The trouble with codetypes: Concerns regarding traditional MMPI-2 interpretative strategies in the medicolegal context. Paper presented at the 5th Annual Conference of the College of Clinical Neuropsychologists. Leura, NSW, Australia, Australian Journal of Psychology 51 (7-10 October 1999), 159.

[13] G. Senior and L. Douglas, Personal injury base rates and interpretative guidelines for the MMPI-2. Special Topic Presentation at the 20th Annual Conference of the National Academy of Neuropsychology. Orlando, Florida, USA, 15-18 November 2000 .

[14] K.R. Vincent, The fragile nature of MMPI code types, Journal of Clinical Psychology 46 (1990), 800-802. 